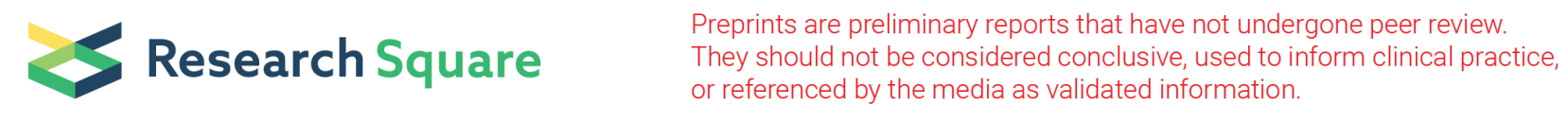

\title{
Effect of microencapsulated Lactobacillus plantarum LIP-1 on cholesterol metabolism in hyperlipidaemic rats
}

\author{
Caiqing Yao \\ Inner Mongolia Agricultural University \\ Wenjing Tian \\ Beijing Vocational College of Agriculture \\ Jiaojiao Song \\ Inner Mongonlia Agricultural University \\ Junguo Wang ( $\sigma$ junguowang@imau.edu.cn )
}

Research article

Keywords: Microencapsulated LIP-1; non-microencapsulated LIP-1 cells; hypolipidaemic effect; lipid metabolism; antioxidative activity

Posted Date: September 23rd, 2019

DOI: https://doi.org/10.21203/rs.2.14790/v1

License: (c) (i) This work is licensed under a Creative Commons Attribution 4.0 International License. Read Full License 


\section{Abstract}

Background: Previous studies have shown that Lactobacillus plantarum LIP-1 has obvious hypolipidemic effect, and microencapsulated probiotics can ensure the strains live through the gastrointestinal tract, although there has been much research on both preparation and assessment methods for probiotics microcapsules, most assessments are made in vitro and few are validated in vivo. In this study, the protective effect of microencapsulation and the possible hypolipidaemic mechanisms of probiotic Lactobacillus plantarum LIP-1 (hereafter LIP-1) were evaluated in rats. Methods: Treatments included rats fed on: normal diet, high-fat diet, high-fat diet with an intragastric supplement of either non-microencapsulated LIP-1 cells (NME LIP-1) or microencapsulated LIP-1 (ME LIP-1). Lipid metabolism indicators were measured during the experiment and following euthanasia. Results: Microencapsulation increased survival and colonization of LIP-1 in the colon. ME LIP-1 was superior to NME LIP-1 in reducing cholesterol. The mechanisms behind the hypolipidemic effect exerted by LIP-1 are possibly due to: promoting the excretion of cholesterol, improving antioxygenic potentials, enhancing recovery from the injury in the liver and intestinal mucosa, promoting the generation of SCFAs, and improving lipid metabolism. Conclusions: This study confirms the role of ME LIP-1 in the prevention and cure of hyperlipidemia and provides theoretical support for the probiotics to enter clinical use. Keywords: Microencapsulated LIP-1; non-microencapsulated LIP-1 cells; hypolipidaemic effect; lipid metabolism; antioxidative activity

\section{Background}

Probiotics are defined as live microorganisms that, when eaten in adequate amounts, confer a health benefit on the consumer [1]. Health and growth benefits offered by probiotics make them an additive with great potential in the food industry. However, there are factors that may influence the functions of probiotics. A key concern is low viability of probiotics in end products owing to adverse conditions during the transportation, storage, marketing and consumption of product; this may mean that they no longer provide the expected health benefits [2]. Factors that influence the stability of probiotics in food include the isolate of probiotic bacterium used, food composition (e.g. moisture content, oxygen availability, $\mathrm{pH}$ and additives), reserve temperature, microbial interactions and the consumer's digestive system (e.g. gastric acid, cholate, enzymes) [2].

Hyperlipidaemia is regarded as a risk factor for cardiovascular disease, which is a leading cause of mortality in many countries [3]. Our research has shown that Lactobacillus plantarum LIP-1 had cholesterol-reducing health benefits, and has been identified as a probiotic isolate from koumiss samples collected in Inner Mongolia [4]. However, for low pH, the tolerance of LIP-1 was limited [4]. Encapsulation has been confirmed as a mean to protect the microorganisms which are part of food products while through the gastrointestinal tract [5]. For this reason, we attempted embedding LIP-1 in milk protein matrices by means of rennet-induced gelation combined with an emulsification technique. Results indicated that when LIP-1 was microencapsulated in this way, it was significantly more resistant to simulated gastric fluid (SGF) in vitro, than free cells [6].

Although there has been much research on both preparation and assessment methods for probiotics microcapsules, most assessments are made in vitro and few are validated in vivo. Considering that the physiological environment within an animal's gastrointestinal tract is more complex than any in vitro simulation, it is important to verify the effect of microencapsulation by in vivo experimentation. The objective of this study was to verify the tolerance of ME LIP-1 to acid conditions in vivo within the digestive system of rats. Specifically, we compared hypolipidaemic symptoms in rats that had been fed either ME LIP-1 or NME LIP-1. Moreover, the possible mechanisms by which LIP-1 might exert positive effects on hypolipidaemic symptoms in vivo were also explored.

\section{Methods}

\section{Preparation of ME LIP-1 and NME LIP-1}

NME LIP-1: LIP-1 was grown in MRS broth (Oxoid Ltd., England) at $37^{\circ} \mathrm{C}$ in an incubator (SANYO Electric Co., Ltd., Japan) for $18 \mathrm{~h}$, during which condition, LIP-1 were subcultured three times and centrifuged at $3000 \mathrm{rpm}$ for $10 \mathrm{~min}$. We discarded the supernatant, washed the cell precipitate for two times with sterilized $0.85 \%(\mathrm{w} / \mathrm{v}) \mathrm{NaCl}$ solution and the final precipitate was resuspended in a cryoprotectant (steriled $10 \%(\mathrm{w} / \mathrm{v})$ skimmed milk and $0.1 \%(\mathrm{w} / \mathrm{v})$ L-monosodium glutamate), and the resulting slurry frozen for $24 \mathrm{~h}$ at $-80^{\circ} \mathrm{C}$ before drying under 90 mTorr vacuum in a freeze dryer (SANYO Electric Co., Ltd., JAPAN) [7]. The main ingredient of the cryoprotectant was similar to the main material of the microcapsule wall to counteract any influence on the results.

ME LIP-1: Encapsulation was according to the method of Tian et al. [6], and the resulting microcapsule slurry was drying in a freeze dryer as same as above. 
Prior to experimentation the viability of LIP-1 as microcapsules or free cells was assessed; $0.5 \mathrm{~g}$ of freeze-dried microcapsules and $0.5 \mathrm{~g}$ of freeze-dried NME LIP-1 powder were suspended in $4.5 \mathrm{~mL}$ of sterile simulated intestinal fluid (SIF: $0.85 \%(\mathrm{w} / \mathrm{v}) \mathrm{NaCl}$ solution with $0.1 \%(\mathrm{w} / \mathrm{v})$ trypsin with a pH of 8.0$)$ and incubated at $37^{\circ} \mathrm{C}$ with constant agitation at $100 \mathrm{rpm}$ for $2 \mathrm{~h}$. The number of viable LIP-1 per gram of freeze-dried microcapsules or free cells was estimated on MRS agar using the plate count method at $37^{\circ} \mathrm{C}$ for $48 \mathrm{~h}$ [8]. Both formulations of LIP-1 were then packaged according to the daily dose (the viability of LIP-1 in microcapsules equal to which in NME LIP1) and stored at $-80^{\circ} \mathrm{C}$ before experimentation.

\section{Animals and feeding regime}

Male Wistar rats (weight, $135 \pm 15 \mathrm{~g}$ ), were purchased from Vital River Lab Animal Technology Co., Ltd (Beijing, China), and housed individually. After a week of acclimatization, all rats were randomly assigned into four groups ( $n=10$ per group) as follows: normal group, model group (hyperlipidaemia model group), control group (LIP-1 group) and experimental group (ME LIP-1 group). The normal group was fed with normal diet throughout the experiment, and other groups were fed with high-fat diet, which consisted of the basic experimental diet $(73.2 \%, \mathrm{w} / \mathrm{w})$, lard $(15.0 \%)$, cholesterol $(1.2 \%)$, custard powder $(10.0 \%)$, sodium taurocholate $(0.3 \%)$ and propylthiouracil (0.3\%) [9]. Individuals in every group also received a daily supplement by intragastric administration $(0.2 \mathrm{~mL}$ supplement/10g body weight) for 4 weeks. For the control and experimental groups this supplement included the LIP-1 treatments suspended in $0.9 \%(\mathrm{w} / \mathrm{v})$ physiological saline; full details are shown in Table 1. Depending on the type of analysis, samples were taken during and at the end of the 4-week experiment when the rats were euthanized. Rats were injected with sodium pentobarbital $(800 \mathrm{mg} / \mathrm{kg})$. Then the cervical spine dislocation method was used to disconnect the rat spinal cord from the brain marrow, causing it to die painlessly[10].

\section{Analyses of faeces}

Faeces were collected every week and the following tests are carried out immediately. Each weekly sample contained all the faeces from the previous week.

Biochemical analyses of faeces: From each sample of faeces $0.2 \mathrm{~g}$ were suspended in $2 \mathrm{ml}$ of sterile saline and centrifuged at 10000 rpm for $10 \mathrm{~min}$. The supernatant was used for $\mathrm{CHO}$ and TG measurement. Analyses were made according to the international standards of the Beijing Sino-UK Institute of Biological Technology, China [11].

Organic acid in faeces: From each sample of faeces $0.1 \mathrm{~g}$ were suspended in $1 \mathrm{~mL}$ ultrapure water, and centrifuged at $10000 \mathrm{rpm}$ for 10 $\min$. To $900 \mu \mathrm{L}$ of the supernatant $15 \mu \mathrm{L}$ of perchloric acid $(60 \%, \mathrm{w} / \mathrm{v})$ were added, then added ultrapure water to $1 \mathrm{~mL}$. After standing for $24 \mathrm{~h}$ at $4^{\circ} \mathrm{C}$ the mixture was filtered through a $0.45 \mu \mathrm{m}$ membrane and subjected to HPLC analysis. The HPLC system was equipped with a multi-wavelength fluorescence detector set at $219 \mathrm{~nm}$. The Agilent Zorbax SB C18 column was maintained at $42^{\circ} \mathrm{C}$ and the degassed mobile phase ( $0.1 \mathrm{M}$ orthophosphoric acid and methanol solution) was used at a flow rate of $0.5 \mathrm{~mL} / \mathrm{min}$ [12].

\section{Biochemical analyses of serum}

After the final feeding treatment, the rats were given no food for $12 \mathrm{~h}$. Blood samples were then collected from the femoral artery, centrifuged for $15 \mathrm{~min}$ at $3500 \mathrm{rpm}$ and the serum (supernatant) removed for TC, TG, HDL, LDL, Apo-A/B, AST, ALT, SOD, GSH-pX and MDA measurement. Analyses were made according to the international standards of the Beijing Sino-UK Institute of Biological Technology, China [11].

\section{Determination of immune organ index}

After rats were euthanized (at 4 weeks by femoral artery puncture followed by immediate exsanguination), the organs of interest (liver, kidney, and spleen) were removed immediately, weighed and washed in cold physiological saline. Organ indices were calculated using the following formula:

Organ index $=$ The weight of the organ $(\mathrm{g}) /$ The weight of the body $(\mathrm{g}) \times 100 \%$. (1)

\section{Biochemical analysis of the liver}

The left lobe of the liver of each rat was homogenized and centrifuged at $3000 \mathrm{rpm}$ for $10 \mathrm{~min}$ and the supernatant used for TC, TG, HDL, LDL, Apo-A/B, SOD, GSH-Px, MDA, LPL and HL measurement. Analyses were made according to the international standards of the Beijing Sino-UK Institute of Biological Technology, China [11].

Page 3/15 


\section{Histological analysis}

Samples of the liver and colon (which were the same section of rats) were fixed with $10 \%(\mathrm{w} / \mathrm{v})$ buffered formalin and embedded in paraffin. Paraffin sections of the liver and colon were stained with Hematoxylin and Eeosin (H \& E) for histopathological examination [13].

\section{Real-time quantitative PCR}

In order to detect the release of ME LIP-1 and the colonization of LIP-1 in the gut, we measured the number of LIP-1 in the colon of rats at the end of the experiment using real-time quantitative PCR (Applied Biosystems, American).

Microbial genomic DNA from $20 \mathrm{mg}$ samples of the colon were extracted using TaKaRa MiniBEST Universal Genomic DNA Extraction Kit (TaKaRa Bio, Code No. 9765, Japan) following the manufacturer's instructions. The DNA concentration was confirmed by absorbance at $260 \mathrm{~nm}$ (A260), and the purity of which was determined by the A260/A280 ratio with a Nanodrop spectrophotometer (Nanodrop Technologies, Wilmington, DE).

Real-time quantitative PCR was done with a Real-Time Quantitative PCR thermocycler (Applied Biosystems, American) using the SYBR Premix Ex Taq ${ }^{\mathrm{TM}} \mathbb{\square}$ (TaKaRa Bio, catalog RR820A, Japan) according to the manufacturer's instructions, and the species-specific primers (forward primer,5'-AAGGCTGAAACTCAAAGG-3'; reverse primer, 5'-AACCCAACATCTCACGAC-3') which we designed referring to Yadav et al. [14]. The thermal cycling conditions used were as follows: an initial DNA denaturation step at $95^{\circ} \mathrm{C}$ for $30 \mathrm{~s}$, followed by 40 cycles of denaturation at $95^{\circ} \mathrm{C}$ for $5 \mathrm{~s}$, primer annealing at $53^{\circ} \mathrm{C}$ for $40 \mathrm{~s}$, extension at $72^{\circ} \mathrm{C}$ for $50 \mathrm{~s}$. Finally, melt curve analysis was performed by slowly cooling the PCRs from 95 to $75^{\circ} \mathrm{C}\left(0.3^{\circ} \mathrm{C}\right.$ per cycle) with simultaneous measurement of the SYBR Green I signal intensity [15].

\section{Statistical analysis}

All statistical analyses were made using SPSS 25.0 software (IBM SPSS Statistics 25 ). Data were expressed as means \pm SD. Data comparisons were made using the Student Newman Keuls test to ensure whether there were significant differences $(P<0.05)$ between treatment means.

\section{Results}

\section{Biochemical analyses of faeces}

Effect of ME LIP-1 treatment on the faecal CHO and TG in rats with hyperlipidaemia: In the model group (high-fat diet), faecal $\mathrm{CHO}$ and TG were significantly higher than the normal group (normal diet) $(15.4 \pm 0.3$ vs $14.5 \pm 0.1 ; 6.0 \pm 0.4$ vs $4.8 \pm 0.2, P<0.05)$, indicating that a large amount of cholesterol is excreted from the body in the faeces when large amounts of fat are consumed (Table 2). Our data showed that when either ME LIP-1 or NME LIP-1 were administered there was significantly higher faecal CHO and TG concentrations compared with the model group (17.2 $\pm 0.3 / 16.5 \pm 0.4$ vs $15.4 \pm 0.3 ; 8.3 \pm 0.6 / 7.4 \pm 1.1$ vs $6.0 \pm 0.4, P<0.05)$. It is possible that LIP-1 may inhibit the absorption of intestinal dietary cholesterol and promote its excretion. Furthermore, this effect was greater when ME LIP-1 was administered compared with NME LIP-1.

Effect of ME LIP-1 treatment on the organic acid content of faeces from rats with hyperlipidaemia: The quantity of lactic acid, acetic acid and propionic acid were significantly lower $(P<0.05)$ in the faeces of rats from the model group compared with the normal group (Table 2). This suggests that hyperlipidaemia may change the in vivo microbial environment, which would affect the production of organic acid. In contrast, the levels of organic acids in the faeces of rats from the groups receiving supplements of ME LIP-1 or NME LIP-1 were significantly higher $(\mathrm{P}<0.05)$ than the model group. This indicates that LIP-1 may promote the generation of organic acids. The levels of organic acid in the faeces of rats fed ME LIP-1 was higher than in faeces from rats fed NME LIP-1, and actually exceeded the levels found in rats from the normal group.

\section{Biochemical analyses of serum}

Effect of ME LIP-1 treatment on serum the lipid levels of rats with hyperlipidaemia: In the model (high-fat) group there was a significant increase in TC, TG, LDL/HDL and APO-B levels and a significant decrease in APO A-l, compared with the normal group $(P<0.05 ;$ Table 3). This indicates that hyperlipidaemia had successfully established in the model treatment and that it could have led to disruption of lipid metabolism in the serum (LDL/HDL: $4.36 \pm 0.46$ vs $0.84 \pm 0.11, P<0.05$ ). Compared to the model group, serum levels of $T C, T G$, 
LDL/HDL and APO-B levels were significantly lower while APO A-I was significantly higher $(\mathrm{P}<0.05)$ compared with the groups supplemented with either ME LIP-1 or NME LIP-1, which indicated that LIP-1 had a significant effect on enhancing lipid metabolism in serum and reducing blood lipid levels in rats with hyperlipidaemia (Table 3). Supplements of ME LIP-1 had a significantly greater effect than supplements of NME LIP-1 $(P<0.05$; Table 3$)$.

Effect of ME LIP-1 treatment on the serum levels of SOD, GSH-Px and MDA in rats with hyperlipidaemia: Compared with the normal group, levels of SOD and GSH-Px were significantly lower (15.81 \pm 0.36 vs $48.54 \pm 1.27 ; 546.87 \pm 43.13$ vs $1542.67 \pm 141.55$, $P<0.05)$, and the level of MDA significantly higher $(6.38 \pm 0.35$ vs $2.38 \pm 0.41, P<0.05)$ in the model group (Table 4), indicating that hyperlipidaemia had suppressed the activity of antioxidant enzymes and increased the level of lipid peroxide in the serum. In the groups supplemented with either ME LIP-1 or NME LIP-1, levels of serum SOD and GSH-Px were significantly higher (33.84 $\pm 1.43 / 24.49 \pm 1.19$ vs $15.81 \pm 0.36 ; 1324.33 \pm 109.91 / 1036.20 \pm 89.23$ vs $546.87 \pm 43.13, P<0.05)$ and the level of serum MDA significantly lower $(2.54 \pm$ $0.07 / 3.68 \pm 0.28$ vs $6.38 \pm 0.35, P<0.05)$ than in the model group, indicating that LIP-1 could enhance the activities of antioxidant enzymes in serum (Table 4). The effect of ME LIP-1 was greater than the effect of NME LIP-1.

Effect of ME LIP-1 treatment on the serum levels of AST and ALT in rats with hyperlipidaemia: Serum levels of AST and ALT were significantly higher in the model group than in the normal group (104.70 \pm 5.10 vs $75.40 \pm 5.53 ; 56.07 \pm 9.43$ vs $36.89 \pm 1.33$, $P<0.05)$, suggesting that hyperlipidaemia damages the liver of rats resulting in increased levels of AST and ALT in the serum (Table 4). In contrast, the content of AST and ALT in serum were significantly lower in the groups receiving supplements of either ME LIP-1 and NME LIP-1 compared with the model (high-fat) group (85.65 $\pm 7.39 / 97.63 \pm 5.95$ vs $104.70 \pm 5.10 ; 42.32 \pm 4.79 / 49.40 \pm 6.22$ vs $56.07 \pm 9.43, P<$ 0.05), although the levels were still higher than that in normal group. This indicates that ME LIP-1 and NME LIP-1 aid recovery from liver injury, but were unable to restore health completely. Moreover, serum levels of AST and ALT were significantly lower $(P<0.05)$ in the experimental group (ME LIP-1) compared with the control group (NME LIP-1), and the serum levels of ALT was no significant difference $(42.32 \pm 4.79$ vs $36.89 \pm 1.33, P>0.05)$ between the experimental group and the normal group (Table 4). This indicates that ME LIP-1 had a better effect on recovery from liver injury than NME LIP-1 (Table 4).

\section{Effect of ME LIP-1 treatment on organ indices}

Organ indices have always been regarded as important in the evaluation of immune function in animals. Research has shown that liver, kidney and spleen indices will be lower than average when animals have an autoimmune disease, thereby indirectly reflecting the health condition of the animal [16]. There was no significant difference $(P>0.05)$ in the kidney and spleen indices amongst all treatment groups indicating that ME LIP-1 and NME LIP-1 had no negative side effects on rats (Table 5). In contrast the liver index was significantly higher in the model (high fat) group compared to the other three groups ( $20.43 \pm 2.86$ vs $16.72 \pm 1.74 / 16.16 \pm 1.72$ / $16.11 \pm 2.13, P<0.05$ ), which may be owing to the high-fat content of the feed accumulating in the liver and increasing the liver index (Table 5). This also indirectly indicates that the model for hyperlipidaemia had established successfully.

\section{Biochemical analyses of the liver}

Effect of the ME LIP-1 treatment on the hepatic TC and TG levels in rats with hyperlipidaemia: There was a trend for suppression of increases in liver TC and TG in the groups of rats receiving either NME LIP-1 or ME LIP-1 compared with the model (high-fat) group (29.9 $\pm 1.4 / 27.5 \pm 0.8$ vs $34.3 \pm 1.6 ; 29.8 \pm 0.1 / 27.0 \pm 1.0$ vs $36.7 \pm 0.5, P<0.05$, Table 6$)$. Furthermore, the levels of liver TC and TG in the rats fed ME LIP-1 were all significantly lower than in the rats fed NME LIP-1 $(P<0.05)$.

Effect of the ME LIP-1 treatment on the hepatic SOD, GSH-Px and MDA in rats with hyperlipidaemia: Variation in SOD, GSH-Px and MDA levels in the liver were consistent with levels in the serum (Table 6), indicating that LIP-1 not only enhanced the activity of antioxidant enzymes in the serum, but also in the liver. Furthermore, the effects of administering ME LIP-1 was greater than the effect of NME LIP-1 (Table 6).

Effect of the ME LIP-1 treatment on hepatic levels of APO A-I, APO-B, LPL and HL in rats with hyperlipidaemia: Hepatic levels of APO A-I, LPL and HL were significantly lower $(P<0.05)$, and levels of APO-B significantly higher $(P<0.05)$ in rats from the model (high-fat) treatment compared with the control (NME LIP-1) and experimental (ME LIP-1) treatment (Table 6). The influence of ME LIP-1 was greater than NME LIP-1. This indicates that hyperlipidaemia caused disruption of lipid metabolism in the liver and that LIP-1 alleviated this, and that ME LIP-1 had the greatest positive effect.

\section{Histological analyses}


No fatty vacuolization was observed in the livers of rats from the normal group (Fig. 1a). However, the hepatocytes of rats from the model (high-fat) group were compressed, and separated by layers of fat and this was accompanied by necrosis and degeneration of hepatic cells (Fig. 1b), indicating that hyperlipidaemia had established successfully in the model group. Supplements of ME LIP-1 and NME LIP-1 substantially suppressed the accumulation of lipid in the liver and repaired the liver cells (Fig. 1c and 1d); Livers of rats receiving the ME LIP-1 supplement were healthier than livers of rats receiving the NME LIP-1, as evidenced by H \& E staining.

From histological analysis of the colon we can see that in the normal group (Fig. 1e), the intestinal villi were long, compact and complete, and the depth of villus crypts and muscle layer thickness were small. However, in the model (high-fat) group (Fig. 1f), the intestinal villi were damaged and had decreased significantly in length; also the lamina propria were loose and the muscle layer was partly incrassated with some inflammation evident, indicating that hyperlipidaemia had damaged the colons of these rats. Compared with the model group, the structure of the intestinal villi was more complete and the edge more compact in the control (NME LIP-1) group (Fig. 1g); in addition, the lamina propria were closer and the muscle layer thickness had decreased, which suggests that LIP-1 has a positive effect on recovery from injury to the colon. The structure of the colons from rats in the experimental (ME LIP-1) group (Fig. 1h) were similar to colons of rats from the normal group except for the length of the intestinal villi. This indicates that ME LIP-1 were better than NME LIP-1 in encouraging recovery from injury to the colon, but did not restore health completely.

\section{Analysis of real-time quantitative PCR}

The results of q-PCR analysis of LIP-1 in the colon (Fig. 2) showed that after treatment with LIP-1 for 28 days, the viable counts of LIP-1 were significantly higher in the experimental (ME LIP-1) group compared with the control (NME LIP-1) group ( $8.20 \pm 0.12$ vs $7.46 \pm 0.33$, $P$ $<0.05$ ), suggesting that microencapsulation significantly increased colonization by LIP-1 in the gut, which may be because the microcapsules provide protection against adverse conditions in the digestive system (e.g. gastric acid, cholate sodium, enzymes), thus enhancing the colonization of LIP-1 in vivo.

\section{Discussion}

Hyperlipidaemia is the main reason of cardiovascular and cerebrovascular diseases, and early prevention and treatment of hyperlipidaemia is necessary to reduce the occurrence of which [17]. Wang et al. [4] showed that LIP-1 had cholesterol-reducing health benefits, but for low pH, the tolerance of LIP-1 was limited, which may weaken its positive effects on hypolipidaemia in vivo. There are also other adverse factors (e.g. gastric acid, bile and enzymes) that can influence the viability of probiotics in vivo [2]. Several studies have demonstrated that encapsulation can provide protection to microorganisms in food products that are subjected to passage through simulated gastrointestinal tracts. Our own studies done in vitro have shown that ME LIP-1 has excellent resistance to simulated gastric fluid (SGF) and wonderful release characteristics in simulated intestinal fluid (SIF) [6]. Regrettably, there are few studies that have verified the effect of microencapsulation on probiotics in vivo.

In this study, we explored the survival of microencapsulated L. plantarum LIP-1 in the host's digestive system and its release under intestinal conditions in vivo using an animal experiment. Ooi and Liong [18] demonstrated that the positive effect of a probiotic was positively correlated with the quantity and activity of viable cells present. In order to illustrate the protective effect of microcapsules for LIP-1, the effect of ME LIP-1 and NME LIP-1 on the regulation of hypolipidaemia in rats were compared, and the colonization of LIP-1 was evaluated by comparing the viable counts of LIP-1 in the colons of rats from the control (NME LIP-1) group and the experimental (ME LIP-1) group. On this basis, possible mechanisms for the effects of LIP-1 on hypolipidaemia were investigated.

It is known that the content of TC, TG, LDL and HDL in serum and the liver are important indicators of lipid levels and, as such, are measured to monitor the effects of strategies to reduce blood fat [19]. Our results showed that using dietary supplements of ME LIP-1 and NME LIP-1 significantly reduced the amount of TC, TG and LDL in the serum and liver of rats being fed a high-fat diet while simultaneously increasing the amount of HDL and faecal CHO, and that the effect was greater for ME LIP-1 than for NME LIP-1. It is also well known that high levels of TC, TG, and LDL in the blood are risk for atherosclerosis, while high level of HDL could reduce the risk of cardiovascular diseases [19]. Therefore, our observations suggested that LIP-1 has a significant positive effect on enhancing lipid metabolism, and that ME LIP-1 was particularly effective. Liver is usually considered to be the main organ to regulate the lipid metabolism and blood lipid levels [20]. Histological analyses of the liver showed that feeding ME LIP-1 to rats on a high-fat diet significantly reduced hepatic lipid deposition compared with rats on the same diet but not receiving the supplement (Fig. 1). These results suggest that the serum levels of TC, TG, and LDL in ME LIP-1-fed rats were certainly reduced, rather than just being redistributed to the liver, which may be achieved by increased excretion. The decreased blood fat may be because the probiotic removed cholesterol by binding it to cellular surfaces and promote the excretion. In previous research, we found that heat-killed cells of LIP-1 showed higher

Page 6/15 
cholesterol removal from media than other strains [4]. Meanwhile, our previous studies have shown that some gut bacteria (namely Bacteroides, Clostridium, Eubacterium, Escherichia and Lactobacillus) were increased in rats given LIP-1 cells, which were known to deconjugate bile salts in the gut and enhance cholesterol excretion [21]. Bao et al. [22] showed similar results for the influence of $L$. plantarum P-8 on lipometabolism in humans. Dvir et al. [23] also found a cholesterol-lowering potential for red microalga, mainly caused by enhanced excretion.

In addition to lipid deposition, we observed other changes to the liver associated with high-fat diets. For example, the hepatocytes of rats in the model (high-fat) group were compressed, separated by layers of fat and suffering from necrosis and degeneration as seen from our histological analysis of the liver. This indicates that hyperlipidaemia damages the liver of rats. In order to investigate the influence of high-fat diets on the liver, as well as the effect of LIP-1 supplements, we measured serum ALT and AST. When there is damage to the liver, the permeability of liver cell membranes is changed and ALT is released into the bloodstream, which causes the increase of ALT in serum. Therefore, serum ALT is a sensitive index of hepatic injury. Furthermore, AST mainly exists in the hepatic mitochondrion and the level could significantly increase when the liver is damaged or seriously necrotic [24]. Higher activities of these enzymes in serum have been found may due to oxidative stress induced by high fat diets [25]. Our results showed that the concentrations of serum ALT and AST did rise significantly in the model (high-fat) group, suggesting that excessive fat intake might cause critical injury to the organ due to the over-production of free radicals and ROS, which exert deleterious effects on liver. And the supplements of ME LIP-1 or NME LIP-1 could significantly decrease AST and ALT levels in the serum. This proves that LIP-1 has a role in recovery from injury of liver, and that ME LIP-1 had a greater effect than NME LIP-1. This was also reflected in the histological analysis of the liver (Fig. 1). This confirms the results of Bao et al. [22] who showed that L. plantarum P-8 was also effective on the prevention of liver injury based on results from ALT and AST analysis.

The oxidative stress is defined as an imbalance between ROS production and the inability of the antioxidant biological system to detoxify free radicals. As a result, the increased level of ROS leads cell to an oxidative stress state, which impacts on a variety of biochemical and physiological processes and is involved in the pathogenesis of many diseases, such as cancer, diabetes and neurodegenerative disorders [26]. MDA is a product of free radicals by oxidizing unsaturated fatty acids, the quantity of which can indirectly reflect the degree of oxidation of body fat. Protective effect against oxidising substances in cells is related to a large number of biological mechanisms, including antioxidant enzyme induction such as SOD and GSH-Px, which could remove the free oxygen radicals [27]. Many studies have shown that hyperlipidaemia could interfere with the balance between production and removal of free radicals, decrease the activity of free radical scavengers, and increase the production of lipid peroxides. In turn the increase in free radicals and lipid peroxide could encourage the occurrence of atherosclerosis and hyperlipidaemia [28]. Some research has shown that oxygen free radicals in serum can promote lipid peroxidation and increase the production of MDA, which could combine with LDL to form MDA-LDL compounds. These compounds could damage vascular endothelial cells and be recognised by mononuclear macrophages and the scavenger receptor of vascular endothelial making them easier to penetrate and resulting in the accumulation of cholesterol in cells. This accumulation of cholesterol turns the cells into foam cells, which is an early indicator of arteriosclerosis [29]. MDA also decreases the activity of enzymes in the mitochondrial membrane, which interferes with the activation of acyl-CoA and the oxidation of fatty acids, leading to accumulation of fat in the liver [30]. Free radicals and lipid peroxides, like MDA, can disrupt lipid metabolism by modification into LDL or HDL and inhibit the activity of cholesterol esterase, which causes an increase in blood lipid abnormalities and cholesterol is more likely to be deposited [31]. Rideout et al. [32] showed that the elimination coefficient of cholesterol reduced by $30 \% \sim 40 \%$ when HDL was modified by lipid peroxides, resulting in an elevation of cholesterol in the blood.

A large number of studies have demonstrated that some lactic acid bacteria possess antioxidative activity, and can decrease the accumulation of free radicals during the digestive process [33]. In our research, ME LIP-1 and NME LIP-1 increased the activity of SOD and GSH-Px in serum and hepatic tissues as expected, decreased the production of MDA and increased the antioxidant ability of the hepatic tissue. ME LIP-1 was more effective than NME LIP-1, preventing the oxidative modification of HDL and LDL and increasing the activity of cholesterol esterase, which resulted in the normal physiological metabolism of cholesterol in the body. In conclusion, LIP-1 could regulate lipid metabolism while removing the oxygen free radicals and increasing the antioxidant ability, which were in response to the decreased level of AST and ALT in serum.

As the liver is the main organ regulating lipid metabolism, any damage could significantly reduce its capacity [20]. For example, APO-B and APO A-I, the important Apolipoproteins in lipid metabolism, are directly affected by damage to the liver. APO-B is the main Apolipoprotein of LDL and APO A-I is the cardio-protective protein in HDL; And high levels of APO-B could cause atherosclerosis, while APO A-I have a good effect on reducing the development of atherosclerosis [34]. Our study clearly demonstrated that the level of APO-B decreased and the APO A-I increased in both the serum and the liver in hyperlipidaemic rats being fed with supplements of ME LIP-1 or 
NME LIP-1, and that ME LIP-1 was more effective than NME LIP-1. Research has shown that the ability to synthetize APO A-I declines when the liver is damaged, and at the same time, the synthesis of transferase is reduced, which promotes the translation from APO A-I precursor to APO A-I, causing the concentration of APO A-I in serum to drop [35]. Given the beneficial effects of LIP-1 on restoration of the liver by scavenging free radicals and increasing antioxidant capacity, it could significantly increase the level of APO A-I and play an important role in regulating the blood lipid metabolism. Malaguarnera et al., [36] showed similar conclusions for the benefit of Bifidobacterium species on steatohepatitis in humans.

LPL and HL are also important enzymes in lipid metabolism. LPL mainly metabolizes CM and VLDL into TG in cells and transfers PHL and APO amongst lipoproteins in the process of lipid metabolism, thus increasing levels of HDL [37]. HL existing on the surface of liver endothelial cells participates in the decomposition of TG in VLDL, LDL and CM, and then promotes the transformation of HDL. In addition, HL could selectively decompose the phospholipid and TG of HDL-2 transforming HDL-2 into HDL-3, which has an inhibitory effect on the formation of atherosclerosis. Therefore, the reduction in activity of LPL and HL gives rise to hyperlipidaemia [38]. Research has shown that the activities of LPL and $\mathrm{HL}$ are regulated by insulin through phosphatidylinositol 3-kinase (PI-3K), and the activity of LPL and HL are reduced when there is either a lack of insulin or insulin resistance [39]. Ma et al., [40] found that high-fat diets could cause insulin resistance, while probiotics could reduce the insulin resistance induced by high-fat diets by increasing the number of hepatic NKT cells and reducing inflammatory signaling. Our results showed that feeding with supplements of ME LIP-1 and NME LIP-1 could significantly increase the activity of LPL and HL, and that ME LIP-1 was more effective than NME LIP-1. We suggest that LIP-1 may reduce insulin resistance and increase the concentration of LPL and $\mathrm{HL}$, thus playing an crucial role in regulating lipoprotein metabolism in the liver, but the specific mechanism require further study. Yu et al. [37] suggested that Sargassum fusiforme affects lipid metabolism in the same way.

Furthermore, SCFAs also contribute to restoration of the colon, and are produced by fermentation of carbohydrate in the colon by bacteria; they are trophic to the small intestinal and colonic mucosa, and stimulate the proliferation of intestinal mucosa epithelial cells and reduce intestinal permeability [41]. Trautwein et al. [42] found that propionate and butyrate provide a source of energy for human colon epithelial cells, and inhibit the growth of pathogenic bacteria and putrefaction bacteria; at the same time, they promote the growth of probiotics (such as Bifidobacterium species, and Lactobacillus species) in the intestine. Marcil et al. [41] found that butyrate can stimulate the intestinal epithelial cells to regulate the absorption and metabolism of lipids, thereby reducing blood cholesterol. Trautwein et al. [42] found that propionate and butyrate can inhibit liver cholesterol synthesis. Furthermore, SCFAs also have other cholesterol-lowering mechanisms. Den et al. [43] demonstrated that SCFAs have the beneficial metabolic effects to protected against high-fat diet-induced obesity and improved insulin sensitivity which were mediated by down-regulation of peroxisome proliferatoractivated receptor-y (PPAR- $\gamma$ ). Our findings agree with those studies. In our study, feeding with supplements of LIP-1 promoted the generation of SCFAs by fermenting dietary carbohydrates, and ME LIP-1 was more effective than NME LIP-1. Possible reason is that the administration of LIP-1 promotes the growth of SCFA-producing strains (like Lactobacillus, Alloprevotella, Eubacterium, Coprococcus and Ruminococcus) which was confirmed by our previous research [21]. Increases in propionic acid can inhibit the synthesis of liver cholesterol and fatty acid, and increases in butyric acid inhibit the absorption of lipids by intestinal epithelial cells, which is an important reason why LIP-1 reduces cholesterol in serum and the liver and increases cholesterol in the faeces. Besides, SCFAs-induced stimulation of insulin sensitivity could increase the activity of LPL and HL, and then promote the lipid metabolism, which confirms our previous conclusion again.

In addition to disruption of blood lipid metabolism, an increase in lipid peroxide can also damage the intestinal mucosa. The colon is an organ that is mainly involved in the digestion and absorption of food, but most importantly the intestinal mucosa is also a biological barrier that could prevent the absorption of harmful materials into the bloodstream. Damage to the colon can influence absorption of nutrients and, above all, increase intestinal permeability, through which inflammatory factors could easily enter the blood, causing inflammatory reactions and disruption of lipid metabolism [44]. It is well know that greater villus height and crypt depth in the colon are indicators that the function of the colon is activated [45]. Our study showed that there were significant differences in villus height, crypt depth and muscle layer thickness between rats from the normal group and the model (high-fat) group, and that the mucous membranes were seriously damaged in the model group. This may be due to increased energy metabolism creating a large number of free radicals after feeding on the high-fat diet; this would cause lipid peroxidation in colon cells, which would damage the secretion glands and mucosal cells [46]. And there may be a reason that high fat diet could increase the level of mucosa-damaging bacteria (like Bilophila and Akkermansia muciniphila) which were confirmed by ljssennagger et al. [47]. LIP-1 reduced the damage caused to the intestinal mucosa by the high-fat diet and improved the health of the colon. The reason for this may be because LIP-1 may improve the antioxygenic potentials and reduce the production of free radicals, thus decreasing the damage to the intestinal mucosa, and increased SCFAs also helpful for the recovering of intestinal mucosa by being trophic and stimulating the proliferation of intestinal mucosa

Page $8 / 15$ 
epithelial cells. Otherwise, Song et al (2017) found a marked decrease in the relative abundances of Bilophila and Akkermansia muciniphila in hyperlipidaemic rats given LIP-1, which could also reduce the damage of intestinal mucosa [21].

This study fully reveals the reasons why LIP-1 reduces blood fat levels in rats with hyperlipidaemia. We have also demonstrated that ME LIP-1 was more effective than NME LIP-1 and the reason for this may be because many LIP-1 cells were destroyed by gastric acids before they reached the intestine, reducing colonisation in the gut. In contrast microcapsules provided protection for LIP-1 against the host's digestive system and released a large number of LIP-1 that were able to colonize the intestines and play a more effective role in the body [18]. The results of the real-time PCR analysis confirmed this hypothesis very well, showing that the viable counts of LIP-1 in the gut were significantly higher in rats fed the ME LIP-1 than in rats fed the NME LIP-1, further indicating that microcapsules provide protection from the digestive system and enhanced colonization in vivo.

\section{Conclusions}

Microencapsulation provides effective protection of LIP-1 in the digestive system. The possible mechanisms for the positive effects of LIP-1 in rats with hypolipidaemia can be elucidated as follows: first, LIP-1 could promote the excretion of cholesterol by binding cholesterol on to cellular surfaces and deconjugate bile salts; second, LIP-1 has the ability to recover the damage of liver and intestinal mucosa by removing oxygen free radicals and improving antioxygenic potentials, and then increase the activity of enzyme or protein (such as LPL, HL and APO A-I) and regulate the lipid metabolism; third, treating with LIP-1 could promote the production of SCFAs, which could inhibit the synthesis of liver cholesterol and fatty acids, reduce the absorption of lipids by intestinal epithelial cells, and promote the lipid metabolism via stimulate the insulin sensitivity and increase the activity of LPL and HL. For clinical application, statins, which are cholesterol-lowering drugs, affect all aspects of the cholesterol profile, but all have been shown to significantly elevate liver enzyme levels [48]. While ME LIP-1 supplementation could provide alternative nutrient supplementation to ameliorate the side effects of statins and has a potential effect on lowering hyperlipidemia.

\section{Abbreviations}

TC, Total cholesterol; TG, Triglyceride; LDL, Low-density lipoprotein; HDL, High-density lipoprotein; APO, Apolipoprotein; APO A-I, Apolipoprotein A-l; APO-B, Apolipoproteins B; MDA, Malondialdehyde; SOD, Superoxide dismutase; CHO, Cholesterol; GSH-Px, Glutathione peroxidase; ALT, Alanine aminotranferease; AST, Aspartate aminotransferase; LPL, Lipoprotein lipase; HL, Hepatic lipase; CM, Chylomicron; VLDL, Very Low Density Lipoprotein; PHL, Phospholipid; ROS, Reactive Oxygen Species; SCFAs, Short-chain fatty acids.

\section{Declarations}

\section{Acknowledgements}

The authors are indebted to the members of the Key Laboratory of Dairy Biotechnology and Engineering, Inner Mongolia Agricultural University, Hohhot, for their technical supports.

\section{Funding}

This work was supported financially by National Natural Science Foundation of China (No. 31660456); and Major Program of Natural Science Foundation of Inner Mongolia (No. 2018ZD14).

\section{Availability of data and materials}

The datasets used and analyzed during the current study available from the corresponding author on reasonable request..

\section{Authors' contributions}

CQY, WJT and JGW designed the experiments. CQY, WJT and JJS performed the experiments. CQY and WJT analyzed the data. CQY and WJT drafted the manuscript. All authors read and approved the final manuscript.

Ethics approval and consent to participate 
This study was approved by the Ethical Committee for Animal Experiments of Inner Mongolia Agricultural University (Permit Number: SYXK(Inner Mongolia)2014-0002). And the animal experiment process strictly abides by the British Animals (Scientific Procedures) Act of 1986 and the European Directive 2010/63/EU.

\section{Competing interests}

No potential conflict of interest was reported by the authors.

\section{Consent for publication}

Not applicable.

\section{Authors' information}

Caiqing Yao and Wenjing Tian contributed equally to the work.

\section{Publisher's Note}

Springer Nature remains neutral with regard to jurisdictional claims in published maps and institutional affiliations.

\section{Author details}

${ }^{1}$ Key Laboratory of Dairy Biotechnology and Engineering, Education Ministry of P. R. China, Department of Food Science and Engineering, Inner Mongolia Agricultural University, Hohhot, 010018, China.

${ }^{2}$ Department of Food and Biological Engineering, Beijing Vocational College of Agriculture, Beijing, 102442, China

\section{References}

[1] Gilliland. WHO expert consultation on evaluation of health and nutritional properties of probiotics in food including powder milk with live lactic acid bacteria. 2001 October 1-4; Córdoba, Argentina.

[2] Islam, Ariful M, Yun CH, Choi YJ, Cho CS. Microencapsulation of live probiotic bacteria. J Microbiol Biotechnol. 2010;20(10):1367-77.

[3] Gao D, Zhu G, Gao Z, Zhiwei L, Liu Z, Wang L, et al. Antioxidative and hypolipidemic effects of lactic acid bacteria from pickled Chinese cabbage. J Med Plants Res. 2011;5(8):1439-46.

[4] Wang J, Zhang H, Chen X, Chen Y, Menghebilige, Bao Q. Selection of potential probiotic lactobacilli for cholesterol-lowering properties and their effect on cholesterol metabolism in rats fed a high-lipid diet. J Dairy Sci. 2012;95(4):1645-54.

[5] Feucht A, Kwak HS. Microencapsulation of lactic acid bacteria (LAB). Korean J Food Sci AN. 2013;33(2):229-38.

[6] Tian W, Song J, Wang Y, Yue L, Wang J, Dan T, et al. Effect of different calcium salts and methods for triggering gelation on the characteristics of microencapsulated Lactobacillus plantarum LIP-1. RSC Adv. 2015;5:73352-62.

[7] Amine KM, Champagne CP, Salmieri S, Britten M, St-Gelais D, Fustier P, et al. Effect of palmitoylated alginate microencapsulation on viability of Bifidobacterium longum during freeze-drying. LWT-Food Sci Technol. 2014;56(1):111-7.

[8] Grosso, Carlos-Raimundo F, Fávaro-T. Stability of free and immobilized Lactobacillus acidophilus and Bifidobacterium lactis in acidified milk and of immobilized B. lactis in yoghurt. Braz J Microbiol. 2004;35(1-2):151-6.

[9] Zhang Y, Du R, Wang L, Zhang H. The antioxidative effects of probiotic Lactobacillus casei Zhang on the hyperlipidemic rats. Eur Food Res Technol. 2010;231(1):151-8.

[10] Zatroch KK, Knight CG, Reimer JN, Pang Daniel SJ. Refinement of intraperitoneal injection of sodium pentobarbital for euthanasia in laboratory rats (Rattus norvegicus). BMC Veterinary Research. 2017;13:60.

[11] Kritchevsky D, Tepper SA. Influence of a fiber mixture on serum and liver lipids and on fecal fat excretion in rats. Nutr Res. 2005;25(5):485-9. 
[12] Baere SD, Eeckhaut V, Steppe M, Maesschalck CD, Backer PD, Immerseel FV, et al. Development of a HPLC-UV method for the quantitative determination of four short-chain fatty acids and lactic acid produced by intestinal bacteria during in vitro fermentation. $J$ Pharmaceut Biomed. 2013;80(3):107-15.

[13] Qin Y, Tian Y. Exploring the molecular mechanisms underlying the potentiation of exogenous growth hormone on alcohol-induced fatty liver diseases in mice. J Transl Med. 2010;8(1):120.

[14] Yadav AK, Tyagi A, Kaushik JK, Saklani AC, Grover S, Batish VK. Role of surface layer collagen binding protein from indigenous Lactobacillus plantarum 91 in adhesion and its anti-adhesion potential against gut pathogen. Microbiol Res. 2013;168(10):639-45.

[15] Livak KJ, Schmittgen TD. Analysis of relative gene expression data using real-time quantitative PCR and the $2^{-\triangle \Delta C T}$ method. Methods. 2001;25(4):402-8.

[16] Padmanabhan M, Arumugam G. Effect of Persea americana (avocado) fruit extract on the level of expression of adiponectin and PPAR-y in rats subjected to experimental hyperlipidemia and obesity. J Complement Integr Med. 2014;11(2):107-19.

[17] Donald M. LJ, David C. G Jr, Neil J. S. Guidelines for cardiovascular risk assessment and cholesterol treatment. JAMA. 2014;311(21):2235.

[18] Ooi LG, Liong MT. Cholesterol-lowering effects of probiotics and prebiotics: a review of in vivo and in vitro findings. Int J Mol Sci. 2010;11(6):2499-522.

[19] Messedi M, Jamoussi K, Frigui M, Laporte F, Turki M, Chaabouni K, et al. Atherogenic lipid profile in Behcet's disease: evidence of alteration of HDL subclasses. Arch Med Res. 2011;42(3):211-18.

[20] Eisenberg S. High density lipoprotein metabolism. J Lipid Res. 1984;25(10):1017-58.

[21] Song J, Tian W, Kwok LY, Wang Y, Shang Y, Menghebilige, et al. Effects of microencapsulated Lactobacillus plantarum LIP-1 on the gut microbiota of hyperlipidaemic rats. Brit J Nutr. 2017;118(7):481-92.

[22] Bao Y, Wang Z, Zhang Y, Zhang J, Wang L, Dong X, et al. Effect of Lactobacillus plantarum P-8 on lipid metabolism in hyperlipidemic rat model. Eur J Lipid Sci Tech. 2012;114(11):1230-36.

[23] Dvir I, Hannah Stark A, Chayoth R, Madar Z, Malis Arad S. Hypocholesterolemic effects of nutraceuticals produced from the red microalga Porphyridium sp. in rats. Nutrients. 2009;1(2):156-67.

[24] Giovanni T, Carmine F, Franco S, Fabrizio P, Franco C, Domenico C, et al. Circulating levels of sirtuin 4, a potential marker of oxidative metabolism, related to coronary artery disease in obese patients suffering from NAFLD, with normal or slightly increased liver enzymes. Oxid Med Cell Long. 2014;2014(2014):920676.

[25] Demori I, Voci A, Fugassa E, Burlando B. Combined effects of high-fat diet and ethanol induce oxidative stress in rat liver. Alcohol. 2006;40(3):185-191.

[26] Gutteridge J, Halliwell B. Free radicals and antioxidants in the year 2000: a historical look to the future. Ann NY Acad Sci. 2000;899(1):136-47.

[27] Liu F, Ng TB. Antioxidative and free radical scavenging activities of selected medicinal herbs. Life Sci. 2000;66(8):725-35.

[28] Sotirios T, Miller YI. Oxidative modification of lipoproteins: mechanisms, role in inflammation and potential clinical applications in cardiovascular disease. Curr Pharm Design. 2011;17(1):27-37.

[29] Králíková E, Ceska R, Rames J. Diet, smoking, and blood lipids in patients with combined familial hyperlipidaemia. Cent Eur J Publ Heal. 1999;7(1):19-23.

[30] Tur Marí JA. The quality of fat: olive oil. Arch Latinoam Nutr. 2004;54(1):59-64.

[31] Fu Q, Liu B. Oxidative modification reduces HDL-induced cholesterol efflux from cultured human arterial smooth muscle cells. Acta Bioch Bioph Sin. 1999;32(3):248-52. 
[32] Rideout TC, Harding SV, Jones PJ, Fan MZ. Guar gum and similar soluble fibers in the regulation of cholesterol metabolism: current understandings and future research priorities. Vasc Health Risk Manag. 2008;4(5):1023-33.

[33] Kuda T, Kaneko N, Yano T, Mori M. Induction of superoxide anion radical scavenging capacity in Japanese white radish juice and milk by Lactobacillus plantarum isolated from aji-narezushi and kaburazushi. Food Chem. 2010;120(2):517-22.

[34] Ahmed K, Rask P, Hurtig-Wennlof A. Serum Apolipoproteins, ApoB/ApoA-I ratio and objectively measured physical activity in the elderly. Scand Cardiovasc J. 2011;45(2):105-11.

[35] Hargrove GM, Junco A, Wong NC. Hormonal regulation of Apolipoprotein Al. J Mol Endocrinol. 1999;22(2):103-11.

[36] Malaguarnera M, Vacante M, Antic T, Giordano M, Chisari G, Acquaviva R, et al. Bifidobacterium longum with fructooligosaccharides in patients with non-alcoholic steatohepatitis. Digest Dis Sci. 2012;57(2):545-53.

[37] Yu Z, Shuai L, Li X, Duan D, Guo Y. Regulating effect of Sargassum fusiforme (Harv.) Setch. on the level of blood lipid in experimental rats with hyperlipidemia. Med Plant. 2013;4(5):54.

[38] Moen CJ, Tholens AP, Voshol PJ, Haan W, Havekes LM, Gargalovic P, et al. The Hyplip2 locus causes hypertriglyceridemia by decreased clearance of triglycerides. J Lipid Res. 2007;48(10):2182-92.

[39] Kim JK, Fillmore JJ, Chen Y, Yu C, Moore IK, Pypaert M, et al. Tissue-specific overexpression of lipoprotein lipase causes tissuespecific insulin resistance. P Natl Acad Sci USA. 2001;98(13):7522-27.

[40] Ma X, Hua J, Li Z. Probiotics improve high fat diet-induced hepatic steatosis and insulin resistance by increasing hepatic NKT cells. J hepatol. 2008;49(5):821-30.

[41] Marcil V, Delvin E, Garofalo C, Levy E. Butyrate impairs lipid transport by inhibiting microsomal triglyceride transfer protein in Caco-2 cells. J Nutr. 2003;133(7):2180-83.

[42] Trautwein EA, Rieckhoff D, Erbersdobler HF. Dietary inulin lowers plasma cholesterol and triacylglycerol and alters biliary bile acid profile in hamsters. J Nutr. 1998;128(11):1937-43.

[43] den Besten G, Bleeker A, Gerding A, van Eunen K, Havinga R, van Dijk TH, et al. Short-chain fatty acids protect against high-fat dietinduced obesity via a PPARy-dependent switch from lipogenesis to fat oxidation. Diabetes. 2015;64(7):2398-408.

[44] Cani PD, Bibiloni R, Knauf C, Waget A, Neyrinck AM, Delzenne NM, et al. Changes in gut microbiota control metabolic endotoxemiainduced inflammation in high-fat diet-induced obesity and diabetes in mice. Diabetes. 2008;57(6):1470-81.

[45] Shamoto K, Yamauchi K. Recovery responses of chick intestinal villus morphology to different refeeding procedures. Poultry Sci. 2000;79(5):718-23.

[46] Hua Z, Han J, Qu D. Effect of different oligosaccharides and peptides on the small intestinal villi histology of mice fed with high-fat diet. Anim Sci Vet Med China. 2012;39(9):123-26.

[47] ljssennagger N, van der Meer R \& van Mil SW. Sulfide as a mucus barrier-breaker in inflammatory bowel disease? Trends Mol Med. 2016;22(3): 190-99.

[48] Chawda HM, Mandavia DR, Parmar PH, Baxi SN, Tripathi CR. Hypolipidemic activity of a hydroalcoholic extract of Cyperus scariosus Linn. root in guinea pigs fed with a high cholesterol diet. Chinese J Nat Med. 2014;12(11): 819-26.

\section{Tables}

Table 1 Description of the treatment groups used in the experiment. 


\begin{tabular}{ccccr}
\hline Groups & Supplement & The number of LIP-1 $(\mathrm{cfu} / \mathrm{mL})$ & Sample Size & Diet \\
\hline normal group & $0.9 \%$ physiological saline & 0 & 10 & normal diet \\
model group & $0.9 \%$ physiological saline & 0 & 10 & high fat diet \\
\hline control group & NME LIP-1 & $2.0 \times 10^{9}$ & 10 & high fat diet \\
\hline experimental group & ME LIP-1 & $2.0 \times 10^{9}$ & 10 & high fat diet \\
\hline
\end{tabular}

Table 2 The effect of microencapsulation of LIP-1 on faecal CHO, TG and organic acids levels in hyperlipidaemic rats ( $\mathrm{n}=10$, mean \pm SD).

\begin{tabular}{|c|c|c|c|c|c|c|}
\hline Groups & $\begin{array}{c}\mathrm{CHO} \\
\left(\mu \mathrm{mol} \cdot \mathrm{g}^{-1}\right)\end{array}$ & $\begin{array}{c}\text { TG } \\
\left(\mu \mathrm{mol} \cdot \mathrm{g}^{-1}\right)\end{array}$ & $\begin{array}{c}\text { lactic acid ( } \mu \mathrm{mol} / g \text { dry } \\
\text { weight) }\end{array}$ & $\begin{array}{l}\text { acetic acid ( } \mu \mathrm{mol} / \mathrm{g} \text { dry } \\
\text { weight) }\end{array}$ & $\begin{array}{c}\text { propionic acid ( } \mu \mathrm{mol} / \mathrm{g} \\
\text { dry weight) }\end{array}$ & $\begin{array}{c}\text { butyric acid ( } \mu \mathrm{mol} / \mathrm{g} \\
\text { dry weight) }\end{array}$ \\
\hline normal group & $14.5 \pm 0.1^{\mathrm{d}}$ & $4.8 \pm 0.2^{\mathrm{d}}$ & $29.78 \pm 6.78^{\mathrm{ab}}$ & $52.72 \pm 8.36^{\mathrm{a}}$ & $14.05 \pm 1.36^{\mathrm{C}}$ & $12.40 \pm 1.58^{\mathrm{c}}$ \\
\hline model group & $15.4 \pm 0.3^{c}$ & $6.0 \pm 0.4^{\mathrm{c}}$ & $17.97 \pm 2.7^{\mathrm{C}}$ & $42.48 \pm 4.33^{b}$ & $12.34 \pm 2.49^{\mathrm{d}}$ & $11.61 \pm 0.98^{\mathrm{c}}$ \\
\hline control group & $16.5 \pm 0.4^{b}$ & $7.4 \pm 1.1^{b}$ & $25.65 \pm 4.89^{b}$ & $49.65 \pm 2.91^{\mathrm{a}}$ & $15.60 \pm 0.45^{\mathrm{b}}$ & $13.74 \pm 1.03^{b}$ \\
\hline $\begin{array}{l}\text { experimental } \\
\text { group }\end{array}$ & $17.2 \pm 0.3^{\mathrm{a}}$ & $8.3 \pm 0.6^{a}$ & $33.47 \pm 5.88^{\mathrm{a}}$ & $51.40 \pm 4.72^{\mathrm{a}}$ & $17.17 \pm 1.39^{\mathrm{a}}$ & $14.89 \pm 1.41^{\mathrm{a}}$ \\
\hline
\end{tabular}

Values in the same column with different superscript letters ( $a, b, c, d)$ differ significantly, $p<0.05$, by one-way ANOVA.

Table 3 The effect of microencapsulation of LIP-1 on serum lipids levels in hyperlipidaemic rats $(n=10$, mean \pm SD).

\begin{tabular}{cccccccc}
\hline Groups & TC $\left(\mathrm{mmol} \cdot \mathrm{L}^{-1}\right) \mathrm{TG}\left(\mathrm{mmol} \cdot \mathrm{L}^{-1}\right)$ & $\mathrm{HDL}\left(\mathrm{mmol} \cdot \mathrm{L}^{-1}\right)$ & $\mathrm{LDL}\left(\mathrm{mmol} \cdot \mathrm{L}^{-1}\right)$ & $\mathrm{LDL} / \mathrm{HDL}$ APO A-I $\left(\mathrm{g} \cdot \mathrm{L}^{-1}\right) \mathrm{APO}-\mathrm{B}\left(\mathrm{g} \cdot \mathrm{L}^{-1}\right)$ \\
\hline normal group & $2.40 \pm 0.02^{\mathrm{d}}$ & $0.40 \pm 0.08^{\mathrm{d}}$ & $1.50 \pm 0.05^{\mathrm{a}}$ & $1.26 \pm 0.12^{\mathrm{d}}$ & $0.84 \pm 0.11^{\mathrm{d}}$ & $1.10 \pm 0.01^{\mathrm{a}}$ & $0.33 \pm 0.01^{\mathrm{c}}$ \\
model group & $6.21 \pm 0.38^{\mathrm{a}}$ & $0.86 \pm 0.11^{\mathrm{a}}$ & $0.92 \pm 0.10^{\mathrm{d}}$ & $3.96 \pm 0.04^{\mathrm{a}}$ & $4.36 \pm 0.46^{\mathrm{a}}$ & $1.02 \pm 0.004^{\mathrm{d}}$ & $0.51 \pm 0.04^{\mathrm{a}}$ \\
\hline control group & $4.95 \pm 0.45^{\mathrm{b}}$ & $0.69 \pm 0.02^{\mathrm{b}}$ & $1.14 \pm 0.03^{\mathrm{c}}$ & $3.54 \pm 0.10^{\mathrm{b}}$ & $3.11 \pm 0.17^{\mathrm{b}}$ & $1.04 \pm 0.0040^{\mathrm{c}}$ & $0.41 \pm 0.02^{\mathrm{b}}$ \\
\hline experimental group & $4.18 \pm 0.03^{\mathrm{c}}$ & $0.53 \pm 0.03^{\mathrm{c}}$ & $1.29 \pm 0.01^{\mathrm{b}}$ & $3.01 \pm 0.06^{\mathrm{c}}$ & $2.32 \pm 1.35^{\mathrm{c}}$ & $1.06 \pm 0.01^{\mathrm{b}}$ & $0.34 \pm 0.04^{\mathrm{c}}$ \\
\hline
\end{tabular}

Values in the same column with different superscript letters ( $a, b, c, d)$ differ significantly, p < 0.05, by one-way ANOVA.

Table 4 The effect of microencapsulation of LIP-1 on the serum levels of SOD, GSH-Px, MDA, AST and ALT in hyperlipidaemic rats ( $\mathrm{n}=10$, mean \pm SD).

\begin{tabular}{ccccccc}
\hline Groups & SOD $\left(\mathrm{U} \cdot \mathrm{ml}^{-1}\right)$ & GSH-PX $\left(\mathrm{U} \cdot \mathrm{ml}^{-1}\right)$ & MDA $\left(\mathrm{nmol} \cdot \mathrm{ml}^{-1}\right)$ & AST $\left(\mathrm{U} \cdot \mathrm{L}^{-1}\right)$ & ALT $\left(\mathrm{U} \cdot \mathrm{L}^{-1}\right)$ & ALT/AST \\
\hline normal group & $48.54 \pm 1.27^{\mathrm{a}}$ & $1542.67 \pm 141.55^{\mathrm{a}}$ & $2.38 \pm 0.41^{\mathrm{c}}$ & $75.40 \pm 5.53^{\mathrm{d}}$ & $36.89 \pm 1.33^{\mathrm{c}}$ & $0.489 \pm 0.02^{\mathrm{c}}$ \\
model group & $15.81 \pm 0.36^{\mathrm{d}}$ & $546.87 \pm 43.13^{\mathrm{d}}$ & $6.38 \pm 0.35^{\mathrm{a}}$ & $104.70 \pm 5.10^{\mathrm{a}}$ & $56.07 \pm 9.43^{\mathrm{a}}$ & $0.536 \pm 0.04^{\mathrm{a}}$ \\
\hline control group & $24.49 \pm 1.19^{\mathrm{c}}$ & $1036.20 \pm 89.23^{\mathrm{c}}$ & $3.68 \pm 0.28^{\mathrm{b}}$ & $97.63 \pm 5.95^{\mathrm{b}}$ & $49.40 \pm 6.22^{\mathrm{b}}$ & $0.502 \pm 0.02^{\mathrm{b}}$ \\
\hline experimental group & $33.84 \pm 1.43^{\mathrm{b}}$ & $1324.33 \pm 109.91^{\mathrm{b}}$ & $2.54 \pm 0.07^{\mathrm{c}}$ & $85.65 \pm 7.39^{\mathrm{c}}$ & $42.32 \pm 4.79^{\mathrm{c}}$ & $0.493 \pm 0.01^{\mathrm{c}}$ \\
\hline
\end{tabular}


Values in the same column with different superscript letters (a, b, c, d) differ significantly, p < 0.05, by one-way ANOVA.

Table 5 The weight of viscera from rats in different groups $(n=10$, mean \pm SD).

\begin{tabular}{cccc}
\hline Groups & Liver & Kidney index $^{\mathrm{B}}$ & Spleen index $^{\mathrm{C}}$ \\
& index $^{\mathrm{A}}$ & & \\
\hline normal group & $16.72 \pm 1.74^{\mathrm{b}}$ & $8.50 \pm 0.70^{\mathrm{a}}$ & $1.74 \pm 0.29^{\mathrm{a}}$ \\
model group & $20.43 \pm 2.86^{\mathrm{a}}$ & $7.74 \pm 0.66^{\mathrm{a}}$ & $1.58 \pm 0.11^{\mathrm{a}}$ \\
\hline control group & $16.16 \pm 1.72^{\mathrm{b}}$ & $7.73 \pm 0.45^{\mathrm{a}}$ & $1.73 \pm 0.18^{\mathrm{a}}$ \\
\hline experimental group & $16.11 \pm 2.13^{\mathrm{b}}$ & $8.18 \pm 0.32^{\mathrm{a}}$ & $1.62 \pm 0.16^{\mathrm{a}}$ \\
\hline
\end{tabular}

Values in the same column with different superscript letters $(\mathrm{a}, \mathrm{b}, \mathrm{c}, \mathrm{d})$ differ significantly, $\mathrm{p}<0.05$, by one-way ANOVA; ${ }^{\mathrm{A}}$ Liver index $=$ The weight of the liver $(\mathrm{g}) /$ The weight of the body $(\mathrm{g}) \times 100 \%{ }^{\mathrm{B}}$ Kidney index $=$ The weight of the kidney $(\mathrm{g}) /$ The weight of the body $(\mathrm{g}) \times 100 \%$; Spleen index $=$ The weight of the spleen $(\mathrm{g}) /$ The weight of the body $(\mathrm{g}) \times 100 \%$.

Table 6 The effect of microencapsulation of LIP-1 on hepatic levels of TC, TG, SOD, GSH-Px, MDA, APO A-I, APO-B, LPL and HL in hyperlipidaemic rats $(\mathrm{n}=10$, mean $\pm \mathrm{SD})$.

\begin{tabular}{|c|c|c|c|c|c|c|c|c|c|}
\hline Groups & $\begin{array}{c}\mathrm{TC} \\
\left(\mu \mathrm{mol} \cdot \mathrm{g}^{-1}\right)\end{array}$ & $\begin{array}{c}\mathrm{TG} \\
\left(\mu \mathrm{mol} \cdot \mathrm{g}^{-1}\right)\end{array}$ & $\begin{array}{c}\text { SOD } \\
\left(\mathrm{U} \cdot \mathrm{mg}^{-1}\right)\end{array}$ & $\begin{array}{l}\text { GSH-PX } \\
\left(\mathrm{U} \cdot \mathrm{mg}^{-1}\right)\end{array}$ & $\begin{array}{c}\text { MDA } \\
\left(\mathrm{nmol} \cdot \mathrm{g}^{-1}\right)\end{array}$ & $\begin{array}{l}\text { APO A-I } \\
\left(\mathrm{mg} \cdot \mathrm{g}^{-1}\right)\end{array}$ & $\begin{array}{l}\text { APO-B } \\
\left(\mathrm{mg} \cdot \mathrm{g}^{-1}\right)\end{array}$ & $\begin{array}{c}\text { LPL } \\
\left(\mu \mathrm{molFFA} \cdot \mathrm{mg}^{-1} \cdot \mathrm{h}\right)\end{array}$ & $\begin{array}{c}\text { HL } \\
\left(\mu \mathrm{molFFA} \cdot \mathrm{mg}^{-1} \cdot \mathrm{h}\right)\end{array}$ \\
\hline $\begin{array}{l}\text { normal } \\
\text { group }\end{array}$ & $26.4 \pm 0.5^{c}$ & $26.7 \pm 2.2^{\mathrm{c}}$ & $9.01 \pm 0.18^{\mathrm{a}}$ & $98.65 \pm 0.36^{\mathrm{a}}$ & $0.55 \pm 0.08^{\mathrm{c}}$ & $52.9 \pm 2.5^{a}$ & $39.5 \pm 4.0^{\mathrm{d}}$ & $0.1799 \pm 0.0073^{a}$ & $0.1615 \pm 0.00284^{\mathrm{a}}$ \\
\hline model group & $34.3 \pm 1.6^{\mathrm{a}}$ & $36.7 \pm 0.5^{\mathrm{a}}$ & $5.97 \pm 0.21^{\mathrm{c}}$ & $66.70 \pm 3.74^{\mathrm{d}}$ & $1.09 \pm 0.04^{\mathrm{a}}$ & $44.5 \pm 1.0^{\mathrm{c}}$ & $80.0 \pm 4.9^{\mathrm{a}}$ & $0.1387 \pm 0.0038^{\mathrm{d}}$ & $0.1092 \pm 0.00626^{\mathrm{d}}$ \\
\hline $\begin{array}{l}\text { control } \\
\text { group }\end{array}$ & $29.9 \pm 1.4^{b}$ & $29.8 \pm 0.1^{b}$ & $7.34 \pm 0.41^{b}$ & $90.40 \pm 1.52^{\mathrm{c}}$ & $0.81 \pm 0.01^{b}$ & $48.9 \pm 1.3^{b}$ & $58.0 \pm 2.0^{\mathrm{b}}$ & $0.1505 \pm 0.0049^{\mathrm{c}}$ & $0.1306 \pm 0.00268^{\mathrm{c}}$ \\
\hline $\begin{array}{l}\text { experimental } \\
\text { group }\end{array}$ & $27.5 \pm 0.8^{c}$ & $27.0 \pm 1.0^{\mathrm{c}}$ & $8.71 \pm 0.12^{\mathrm{a}}$ & $94.82 \pm 0.28^{b}$ & $0.63 \pm 0.12^{\mathrm{c}}$ & $49.7 \pm 0.6^{b}$ & $48.9 \pm 4.0^{\mathrm{c}}$ & $0.1649 \pm 0.0060^{\mathrm{b}}$ & $0.142 \pm 0.00529^{b}$ \\
\hline
\end{tabular}

Values in the same column with different superscript letters (a, b, c, d) differ significantly, p < 0.05, by one-way ANOVA.

\section{Figures}



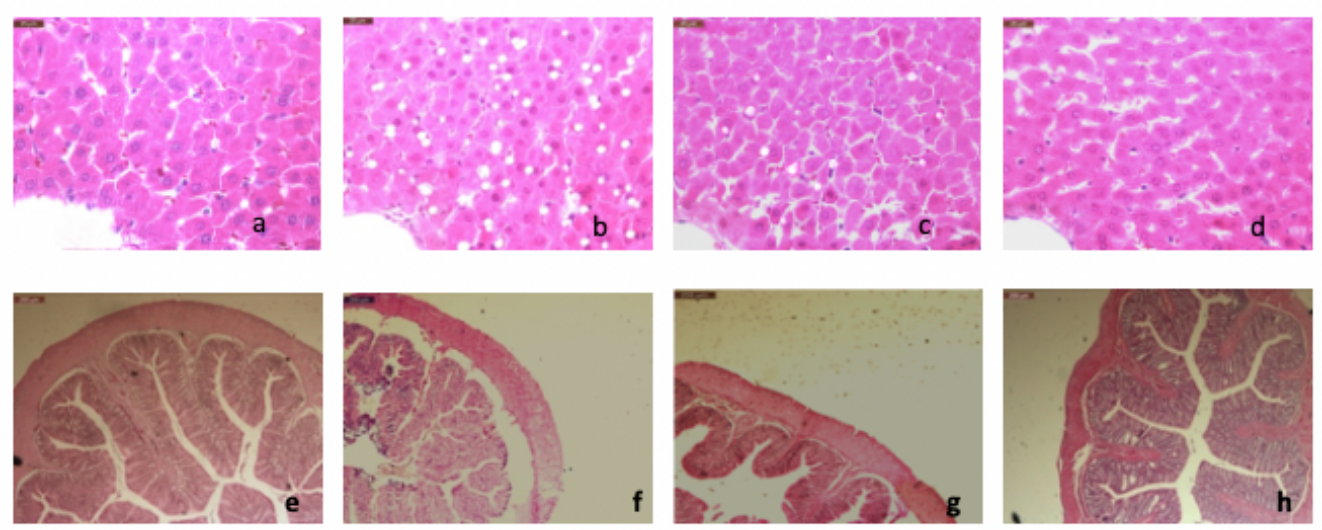

Figure 1

The effect of microencapsulation of LIP-1 on liver and colon: (a) hepatic lipid deposition in the normal group (bar =25 $\mu \mathrm{m}$ ); (b) hepatic lipid deposition in the model group (bar =25 $\mu \mathrm{m}$ ); (c) hepatic lipid deposition in the control group (bar =25 $\mu \mathrm{m})$; (d) hepatic lipid deposition in the experimental group (bar $=25 \mu \mathrm{m})$; (e) histological analysis of the colon in the normal group $(\mathrm{bar}=250 \mu \mathrm{m}) ;(\mathrm{f})$ histological analysis of the colon in the model group (bar =250 $\mu \mathrm{m})$; (g) histological analysis of the colon in the control group (bar $=250$ $\mu \mathrm{m})$; (h) histological analysis of the colon in the experimental group $(\mathrm{bar}=250 \mu \mathrm{m})$.

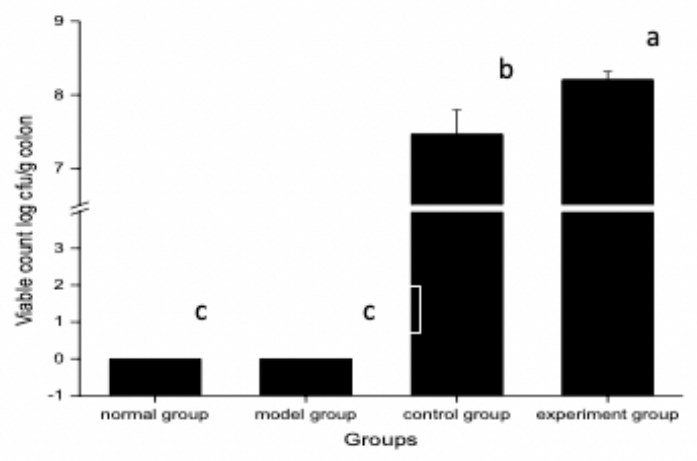

\section{Figure 2}

The effect of microencapsulation of LIP-1 on viable counts of LIP-1 from colon samples in hyperlipidaemic rats $(n=10, m e a n \pm S D)$. Values in the same index with different superscript letters $(a, b, c)$ differ significantly, $p<0.05$, by one-way ANOVA.

\section{Supplementary Files}

This is a list of supplementary files associated with this preprint. Click to download.

- Highlights.docx

- GraphicalAbstract.jpg

- NC3RsARRIVEGuidelinesChecklist2014.docx 